\title{
Liver Resection Without Total Vascular Exclusion: Hazardous or Beneficial?
}

\author{
An Analysis of Our Experience
}

\author{
Guido Torzilli, MD, PhD, FPCR, Masatoshi Makuuchi, MD, PhD, Yutaka Midorikawa, MD, Keji Sano, MD, \\ Kazuto Inoue, MD, PhD, Tadatoshi Takayama, MD, PhD, and Keiichi Kubota, MD, PhD
}

From the Hepato-Biliary-Pancreatic Surgery Division, Artificial Organ and Transplantation Division, Department of Surgery, Graduate School of Medicine, University of Tokyo, Japan

\section{Objective}

To evaluate retrospectively the safety and radicality of liver resection performed without total vascular exclusion (TVE).

\section{Summary Background Data}

TVE is recommended for safe liver surgery, at least in the case of resection of the paracaval portion of the liver. However, it has some drawbacks because of its invasiveness.

\section{Methods}

The authors retrospectively evaluated 329 of 471 consecutive patients who underwent liver resection from October 1994 to October 1999. All of these patients had tumors involving segments 1,7 , or 8 or the cranial portion of segment 4 , or underwent major hepatectomies that required exposure of the inferior vena cava (IVC), the main trunks of the hepatic veins, or both.
Sixty-four patients underwent resection that included segment 1, with or without the reconstruction of the IVC, the hepatic vein, or both.

\section{Results}

Three hundred twenty-four of 329 procedures were done under intermittent warm ischemia; no clamping methods were used in 6. TVE was never needed. There were no postoperative 30 -day deaths. The complication rate was $25.5 \%$, and only $2.1 \%$ had major complications. Only 13 (3.9\%) patients required whole blood transfusion. Part of the wall of the IVC was resected in six patients, and the hepatic veins were reconstructed in four. Surgical clearance was achieved in all patients undergoing surgery for a tumor.

\section{Conclusions}

These results show that liver surgery performed without TVE is safe and effective even in aggressive procedures for liver tumors involving the cavohepatic junction. Therefore, TVE should be further restricted to exceptional patients.
Limiting intraoperative blood loss and the consequent need for whole blood transfusion is a widely accepted goal in liver resection, ${ }^{1,2}$ because these factors seem to impair the patient's outcome ${ }^{3}$ and prognosis. ${ }^{4}$ To achieve this goal,

Supported in part by grants-in-aid for Cancer Research from the Ministry of Health and Welfare of Japan.

Correspondence: Guido Torzilli, MD, PhD, FPCR, Via Lodovico il Moro, 87, 20143 Milano, Italy. E-mail: torzillg@tin.it or torvalgc@tin.it. Reprints: Masatoshi Makuuchi, MD, PhD, Professor and Chairman, Hepato-Biliary-Pancreatic Surgery Division, Artificial Organ and Transplantation Division, Department of Surgery, Graduate School of Medicine, University of Tokyo, 7-3-1 Hongo Bunkyo-ku, Tokyo 1138655, Japan.

E-mail: makuuchi-tky@umin.ac.jp

Accepted for publication July 12, 2000. liver resection performed under intermittent warm ischemia may be useful. However, there is controversy regarding the extent and method for obtaining warm ischemia. Intermittent or continuous portal triad clamping, hemihepatic vascular occlusion, and total vascular exclusion (TVE) with or without aortic clamping are the most commonly used methods. TVE was devised to allow bloodless major hepatectomies, ${ }^{5}$ and it is now recommended for patients with tumors in the paracaval portion of the liver. ${ }^{6,7}$ However, it has some drawbacks because of its invasiveness and related hemodynamic changes. Our policy includes intermittent warm ischemia accomplished by portal triad clamping and hemihepatic vascular occlusion. We reviewed our series of hepatectomies with regard to safety, because all were accomplished without TVE. We focused our analysis on pa- 
tients in whom TVE is generally recommended, such as those with tumors in the posterosuperior segments of the liver, or in whom TVE is considered strictly indicated, such as those with a tumor in the paracaval portion of the liver. Further, to elucidate the need for TVE in patients who require resection of the paracaval portion of the liver, we compared their postoperative outcomes with those of other patients.

\section{METHODS}

Inclusion criteria for this study were based on the tumor location and the type of resection, with major hepatectomy defined as the removal of at least one Couinaud sector, 8 segmentectomy as systematic subsegmentectomy described previously, ${ }^{9}$ and limited resection as nonanatomical tumor removal. According to these criteria, we selected 329 of 471 consecutive patients who underwent liver resection at Tokyo University Hospital from October 1994 to October 1999. All these patients had tumors involving segments 1,7 , or 8 or the cranial portion of segment 4 , or underwent major hepatectomies that required exposure of the inferior vena cava (IVC), the main trunks of the hepatic veins, or both. The 142 excluded patients were those in whom the tumors involved exclusively segments $2,3,5$, or 6 or just the caudal portion of segment 4 , and who did not undergo major resections.

There were 228 male patients and 101 female patients $(\mathrm{M}: \mathrm{F}=2.3: 1$ ), and the mean age was 62 years (median 63, range 13-89). Patients were selected for surgery as previously reported ${ }^{10,11}$ based on the presence or absence of ascites, total serum bilirubin level, and indocyanine green retention rate at 15 minutes $\left(\mathrm{ICG}_{15}\right)$. The preoperative mean total serum bilirubin level was $0.8 \mathrm{mg} / \mathrm{dL}$ (median 0.6, range $0.3-5$ ), and the mean $\mathrm{ICG}_{15}$ was $13.5 \%$ (median 11.8 , range 1-49). There were $38 \mathrm{HBsAg}$ carriers and $126 \mathrm{HCV}-$ $\mathrm{Ab}$-positive patients. Patients were grouped according to the presence (group A) or absence (group B) of tumor involvement of segment 1 , the hepatic vein, the IVC, or both. There were 64 patients in group A and 265 in group B. Table 1 shows the background liver status and the indications for surgery for the whole series and the two groups.

\section{Statistical Analysis}

A bilateral Student $t$ test for unpaired data were used to compare the age, $\mathrm{ICG}_{15}$, tumor size, tumor number, mean intraoperative blood loss, clamping time, operative time, and postoperative hospital stay in the two groups. The chi-square test was used to compare the aforementioned groups in terms of background liver and type of surgery and to compare their complication rates. $P$ value was set at .05 .

\section{RESULTS}

Major hepatectomies were performed in 136 patients, segmentectomy was performed in 96 (multiple segmentec-
Table 1. HISTOLOGY

\begin{tabular}{lc}
\hline \multicolumn{1}{c}{ Histology of the Liver } & $\begin{array}{c}\text { Number of } \\
\text { Patients (\%) }\end{array}$ \\
\hline Cirrhotic & $135(41)$ \\
Not cirrhotic & $194(59)$ \\
\hline \multicolumn{1}{c}{ Histology of the Tumor } & \\
\hline HCC & $210(63.8)$ \\
Metastases & $66(20)$ \\
Hilar bile duct cancer & $17(5.2)$ \\
Intrahepatic CCC & $10(3.3)$ \\
Mixed HCC and CCC & $5(1.6)$ \\
Hemangioma & $5(1.6)$ \\
Intrahepatic bile duct stone & $3(0.9)$ \\
Gallbladder carcinoma & $2(0.5)$ \\
Benign biliary strictures & $2(0.5)$ \\
Inflammatory pseudotumors & $2(0.5)$ \\
Stromal tumor & $1(0.3)$ \\
HCC and hilar bile duct cancer & $1(0.3)$ \\
HCC and gallbladder cancer & $1(0.3)$ \\
HCC and hemangioma & $1(0.3)$ \\
Hilar bile duct cancer and gallbladder cancer & $1(0.3)$ \\
Angiomyolipoma & $1(0.3)$ \\
Intrahepatic arterioportal shunt & $1(0.3)$ \\
\end{tabular}

CCC, cholangiocarcinoma; HCC, hepatocellular carcinoma.

tomy in 31), and limited resections were performed in 97 (multiple in 56). Limited resections were associated with segmentectomies or major hepatectomies in 49 patients; other organs or structures were also resected in 35 patients. Table 2 shows the surgical procedures in detail. All the procedures were macroscopically radical.

There were no postoperative 30-day deaths, and all the patients returned to their normal daily life. The complication rate was $25.5 \%$, and major complications, defined as those requiring additional surgery, occurred in only seven patients $(2.1 \%)$. The types of complications and their distribution between the two groups are shown in Table 3.

The mean operative time was 442 minutes (median 405, range 125-1,065). Mean blood loss was $853 \mathrm{~mL}$ (median 690 , range 61-4,072), and 13 patients were transfused with whole blood $(3.9 \%)$. Only two patients in group A $(3.1 \%)$ received whole blood transfusion. The Pringle maneuver was performed in 285 patients, hemivascular occlusion was carried out in 38 , and warm ischemia was not accomplished in 6. TVE was never considered necessary. Mean clamping time was 69 minutes (median 60, range 0-248).

The comparison between groups $\mathrm{A}$ and $\mathrm{B}$ is shown in Table 4. There were no statistically significant differences in terms of age, intraoperative blood loss, clamping time, complication rates, or background liver status between the two groups. Although this difference was not statistically significant, the incidence of major resection tended to be higher in the patients in group A, who had significantly longer operations and a longer postoperative hospital stay. 
Table 2. SURGICAL PROCEDURES

\begin{tabular}{lc}
\hline \multicolumn{1}{c}{ Type of Surgery } & $\begin{array}{c}\text { Number of } \\
\text { Patients }\end{array}$ \\
\hline Major resections & 136 \\
Left hepatectomy & 26 \\
Extended left hepatectomy & 26 \\
Left trisectoriectomy & 1 \\
Right hepatectomy & 26 \\
Extended right hepatectomy & 28 \\
Right hepatectomy and segment 4 & 2 \\
resection & 13 \\
Right paramedian sectoriectomy & 3 \\
Extended right paramedian sectoriectomy & 12 \\
Right lateral sectoriectomy & 5 \\
Extended right lateral sectoriectomy & 3 \\
Central bisectoriectomy & 1 \\
Segment 2 and 3 resection* & 96 \\
Segmentectomies & 97 \\
Limited resections & 41 \\
Single & 56 \\
Multiple & 329 \\
Total & \\
to his exclusion from this study, also underwent multiple limited resection and \\
reconstruction of the middle hepatic vein. & \\
\hline & \\
&
\end{tabular}

Hepatic vein reconstruction was performed in four patients. In one patient who underwent extended right hepatectomy for liver metastases from colorectal cancer, the middle hepatic vein was reconstructed because of congestion of the caudal portion of the remnant segment 4 (Fig. 1). This reconstruction was done using a vein graft interposition obtained by skeletonizing a tumor-free tract of the right hepatic vein prepared at the back table from the resected specimen. In the second patient, part of the anterior wall of the right hepatic vein was resected because of infiltration by colorectal liver metastases (Fig. 2). The vessel was reconstructed by patch closure using a vein graft obtained from the right ovarian vein. At that time, this patient underwent simultaneous and radical removal of 17 metastatic lesions. In the third patient, 12 colorectal liver metastases were removed by segmentectomy of segments 2 and 3 and multiple limited resections in segments $4,5,6$, and 7. During this procedure, a portion of the wall of the middle hepatic vein was removed because of infiltration by the tumor in segment 4 (Fig. 3), and the vessel was reconstructed by direct suture. The fourth patient underwent reconstruction of both the right and middle hepatic veins. This patient, a 37-year-old man, had two colorectal liver metastases: one was in segments 3 and 4 and infiltrated the middle hepatic vein, and the other occupied part of segments 5, 6, 7, and 8 and infiltrated part of the anterior wall of the right hepatic vein (Fig. 4). He underwent a left hepatectomy with resection of the main trunk of the middle hepatic vein and limited resection of the tumor in the right segments with removal of part of the anterior wall of the right hepatic vein. The middle
Table 3. COMPLICATIONS

\begin{tabular}{|c|c|c|c|}
\hline \multirow[b]{2}{*}{ Complication } & \multicolumn{3}{|c|}{ Group } \\
\hline & $\begin{array}{c}\text { Overall } \\
\text { (329 } \\
\text { pts) }\end{array}$ & $\begin{array}{c}\text { A } \\
\text { (64 } \\
\text { pts) }\end{array}$ & $\begin{array}{c}\text { Group B } \\
\text { (265 } \\
\text { pts) }\end{array}$ \\
\hline \multicolumn{4}{|l|}{ Major } \\
\hline Patients & 7 & 4 & 3 \\
\hline Number of episodes & 8 & 5 & 3 \\
\hline \multicolumn{4}{|l|}{ Type } \\
\hline Abdominal abscess & 3 & 2 & 1 \\
\hline Hemorrhage & 2 & 2 & - \\
\hline Splenic vein thrombosis & 1 & - & 1 \\
\hline Bilioenteric anastomotic dehiscence & 1 & 1 & - \\
\hline Pneumothorax & 1 & - & 1 \\
\hline \multicolumn{4}{|l|}{ Minor } \\
\hline Patients & 77 & 17 & 60 \\
\hline Number of episodes & 80 & 20 & 64 \\
\hline \multicolumn{4}{|l|}{ Type } \\
\hline Pleural effusion & 36 & 9 & 27 \\
\hline Bile leak & 18 & 4 & 14 \\
\hline Wound infection & 6 & 2 & 4 \\
\hline Ascites & 5 & 1 & 4 \\
\hline Atelectasis & 5 & 1 & 4 \\
\hline Biliary fistula & 4 & - & 4 \\
\hline Dynamic ileus & 2 & - & 2 \\
\hline Cholangitis & 2 & 2 & - \\
\hline Wound dehiscence & 1 & - & 1 \\
\hline Hyperamylasemia & 1 & 1 & - \\
\hline \multicolumn{4}{|l|}{ Total } \\
\hline Patients & 84 & 21 & 63 \\
\hline Number of episodes & 92 & 25 & 67 \\
\hline
\end{tabular}

hepatic vein was reconstructed using the left hepatic vein. For this purpose, the left hepatic vein was previously skeletonized in situ, and its two distal branches were then sutured, resulting in a single-lumen stump with a diameter that matched that of the stump of the middle hepatic vein on the liver cut surface. The left hepatic vein was then shifted to the right and anastomosed with the aforementioned stump of the middle hepatic vein. Reconstruction of the right hepatic vein was done by patch closure using a portion of the wall of the umbilical tract of the left portal vein prepared at the back table from the resected specimen.

The IVC was reconstructed in six patients. The type of vascular reconstruction was based on the estimated residual diameter of the vessel. In one patient, we used a patch graft obtained by dissecting a portion of the wall of the right hepatic vein, which was prepared at the back table from a tumor-free portion of the resected liver. One patient had recurrent colorectal liver metastases in the paracaval portion that infiltrated the IVC to more than two thirds of the circumference of the vessel (Fig. 5). In this patient, limited resection of the tumor was accomplished, but because of the wide infiltration of the IVC, it was necessary to clamp this vessel at the prehepatic portion and infrahepatically just below the confluence of the hepatic veins. The IVC was then reconstructed with a patch graft closure using GoreTex (Flagstaff, AZ) and remained clamped for 57 minutes. 
Table 4. COMPARISON OF THE TWO GROUPS

\begin{tabular}{|c|c|c|c|}
\hline Factor & Group A & Group B & $\begin{array}{c}P \\
\text { Value }\end{array}$ \\
\hline Age (years) & $64(65 ; 33-81)$ & $61(63 ; 13-89)$ & .07 \\
\hline $\mathrm{ICG}_{15}(\%)$ & $12(9 ; 1-42.5)$ & $13.9(12 ; 2-49)$ & .15 \\
\hline Tumor size (cm) & $4(3 ; 1-12)$ & 5 (4; 0.5-22) & .03 \\
\hline Tumor number & $2(1 ; 1-19)$ & $2(1 ; 1-22)$ & .32 \\
\hline Intraoperative blood loss (mL) & $963(800 ; 195-3136)$ & $827(654 ; 61-4072)$ & .13 \\
\hline Inflow clamping time (min) & $71(60 ; 15-248)$ & $68(60 ; 0-207)$ & .64 \\
\hline Operative time (min) & $566(565 ; 244-1065)$ & $412(390 ; 125-910)$ & $<.01$ \\
\hline Postoperative hospital stay (days) & $27(21 ; 11-80)$ & $21(19 ; 10-47)$ & .01 \\
\hline Complications & $21(32.8 \%)$ & $63(23.8 \%)$ & .5 \\
\hline \multicolumn{4}{|l|}{ Type of resection } \\
\hline Major & $36(56.2 \%)$ & $100(37.7 \%)$ & .8 \\
\hline Segmentectomy & $7(10.9 \%)$ & $89(33.6 \%)$ & .9 \\
\hline Limited & $21(32.8 \%)$ & $76(28.7 \%)$ & .4 \\
\hline \multicolumn{4}{|l|}{ Background liver } \\
\hline Cirrhotic & $40(62 \%)$ & $95(36 \%)$ & .9 \\
\hline Not cirrhotic & $24(38 \%)$ & $170(64 \%)$ & \\
\hline
\end{tabular}

$I C G_{15}$, indocyanine green retention rate at 15 minutes. For the factors age, ICG ${ }_{15}$, tumor size, tumor number, intraoperative blood loss, clamping time, operative time, and postoperative hospital stay, the values correspond to the mean (median; range). For the factors complications, type of resection, and background liver, the values indicate the number of patients (percentage).

During this period, liver perfusion was maintained with outflow of the hepatic veins. Liver dissection was performed simply under the Pringle maneuver (clamping time 123 minutes). In the remaining four patients, the IVC was directly sutured (Figs. 6 and 7).

None of the patients who underwent IVC or hepatic vein reconstruction received blood transfusion.

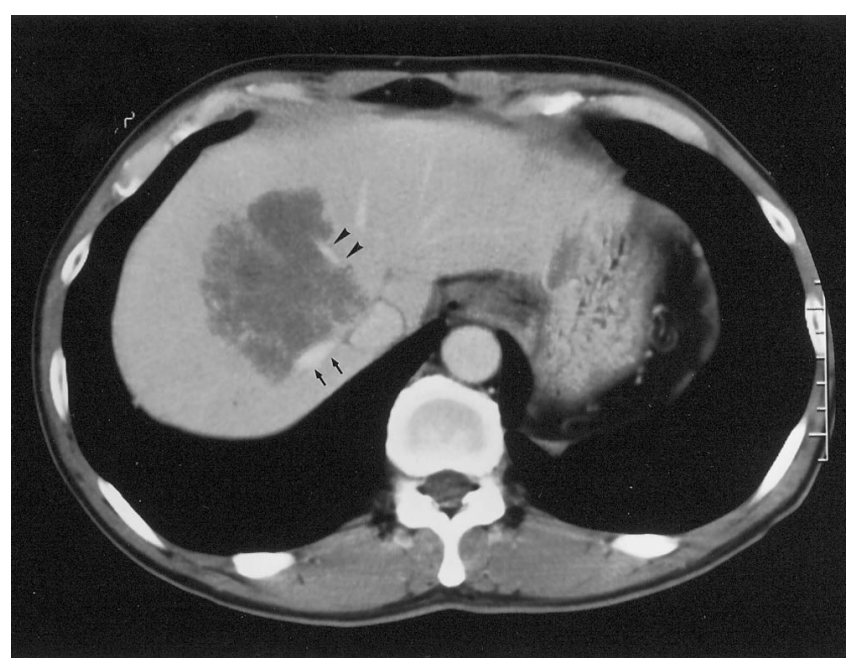

Figure 1. Computed tomography scan shows a liver metastasis from a colorectal cancer that infiltrates the right hepatic vein (arrows) and involves the middle hepatic vein (arrowheads). This patient underwent extended right hepatectomy, and the middle hepatic vein was reconstructed because of congestion of the caudal portion of the remnant segment 4. Reconstruction was performed using a vein graft interposition obtained by skeletonizing a tumor-free tract of the right hepatic vein prepared at the back table from the resected specimen.

\section{DISCUSSION}

To limit blood loss, vascular clamping is a commonly accepted method in liver surgery. For this purpose, the Pringle maneuver ${ }^{12}$ is the most widely adopted technique, and it has been shown to be safe and well tolerated. Recently Man et $\mathrm{al}^{13}$ showed in a prospective randomized trial that the postoperative outcome of patients who underwent liver resection with the Pringle maneuver was better than that of those who underwent surgery without it. Intermittent

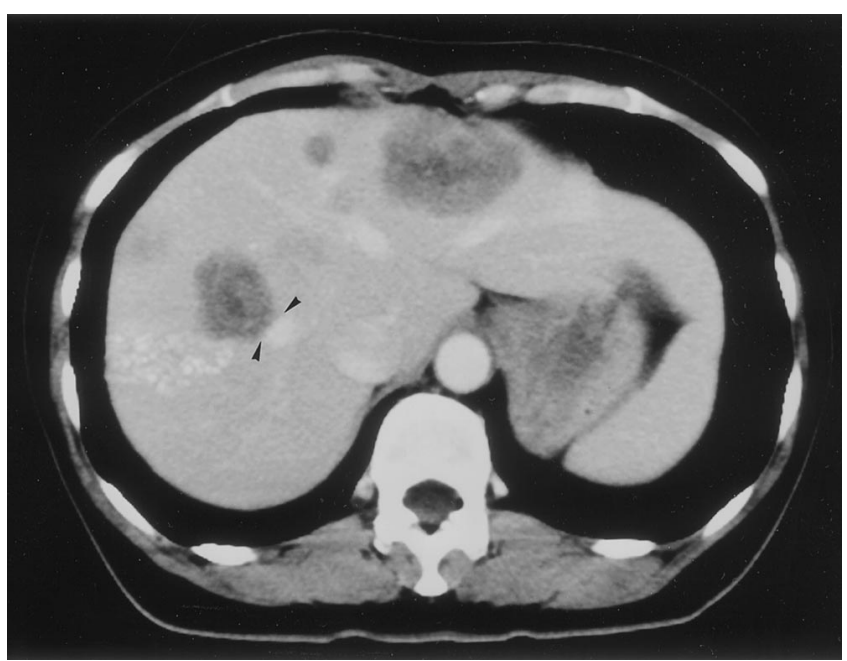

Figure 2. Computed tomography scan shows the anterior wall of the right hepatic vein in direct contact with 1 of 17 colorectal liver metastases (arrowheads). This patient underwent simultaneous and radical removal of all the metastatic lesions. The vessel was reconstructed by patch closure using a vein graft from the right ovarian vein. 


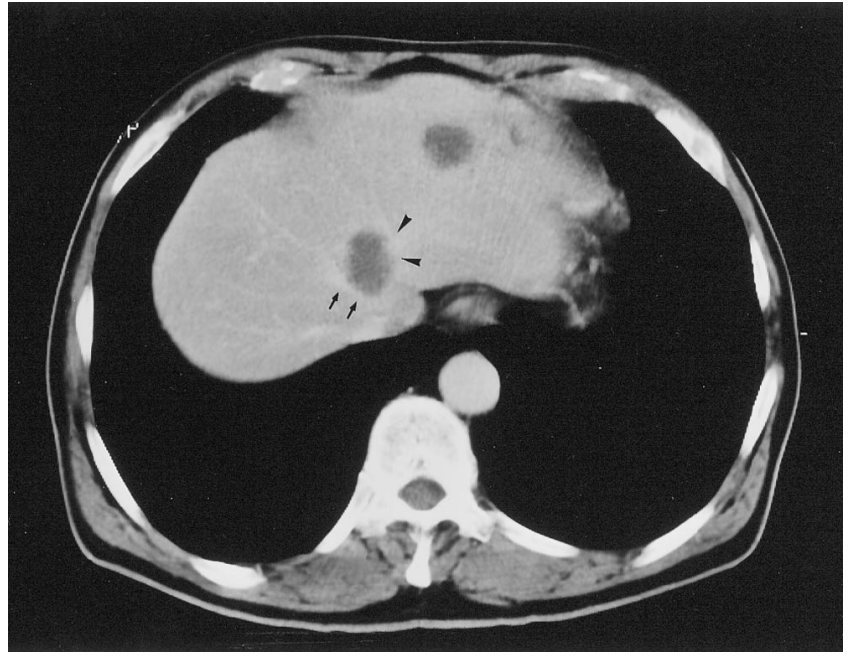

Figure 3. In this patient, who had 12 colorectal liver metastases, the computed tomography scan shows a lesion in segment 4 that appeared to be in direct contact with the middle (arrows) and left hepatic veins (arrowheads). During surgery, although it was possible to separate the lesion from the wall of the left hepatic vein, which was not infiltrated, part of the wall of the middle hepatic vein was removed because of tumor involvement. The vessel was reconstructed by direct suture.

portal triad clamping seems to be safe up to 2 hours $^{14}$ and can also be carried out safely in patients with cirrhosis. ${ }^{15-17}$ Belghiti et $\mathrm{al}^{18}$ recently demonstrated that intermittent clamping is better tolerated than continuous clamping.
The hemihepatic vascular occlusion obtained with hilar dissection requires care and skill by the surgeon, who must know perfectly the specific anatomy of the region. Some authors have proposed the use of color Doppler sonography ${ }^{19}$ or the en masse technique ${ }^{20-22}$ to avoid hilar dissection. However, a preoperative study that includes angiography and careful hilar dissection should help the surgeon to avoid major accidental injuries, and conversely hilar dissection performed properly rules out the risk of damaging the hilar structures with blind maneuvers. Other adjunctive techniques to reduce intraoperative bleeding such as ischemia induced by portal triad clamping and cooling the liver in vivo ${ }^{23}$ or further modifications of the Pringle maneuver ${ }^{24}$ have also been proposed. All these techniques have been thought to combine the need to limit blood loss with reductions in the presumptive side effects of the Pringle maneuver, such as bowel congestion and pancreatic damage. ${ }^{25,26}$ However, despite this trend, the most widespread technique, other than portal triad clamping, is paradoxically more invasive: TVE.

First proposed by Heaney et $\mathrm{al}^{27}$ and further developed by other authors, ${ }^{5,28-30}$ this technique involves two principal maneuvers. One includes supraceliac aortic clamping, ${ }^{31}$ and in the other only the portal triad and the infrahepatic and suprahepatic IVC are clamped. Heaney et al initially applied TVE with supraceliac aortic clamping experimentally in dogs, and this was the first attempt at liver resection under TVE. Its major drawback is its major invasiveness and
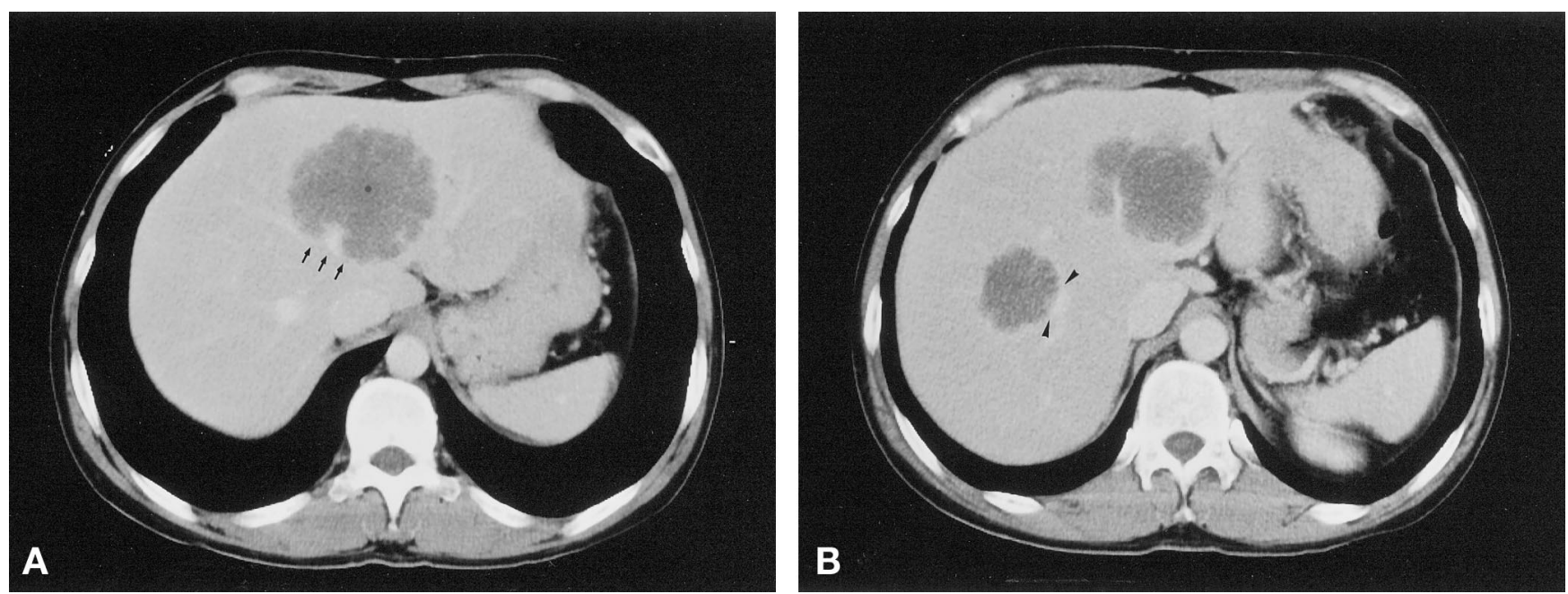

Figure 4. Computed tomography scans show two colorectal liver metastases: one was in segments 3 and 4 and infiltrated (arrows) the middle hepatic vein (A), and the other occupied part of segments 5, 6, 7, and 8 and infiltrated part of the anterior wall of the right hepatic vein (arrowheads) (B). The patient underwent a left hepatectomy with resection of the main trunk of the middle hepatic vein and limited resection of the tumor in the right segments, with removal of part of the anterior wall of the right hepatic vein. The middle hepatic vein was reconstructed using the left hepatic vein. For this purpose, the left hepatic vein was skeletonized in situ, and its two distal branches were then sutured to give a single-lumen stump with a diameter matching that of the stump of the middle hepatic vein on the liver cut surface. The left hepatic vein was then shifted to the right and anastomosed with the aforementioned stump of the middle hepatic vein. The right hepatic vein was reconstructed by patch closure using a portion of the wall of the umbilical tract of the left portal vein prepared at the back table from the resected specimen. 


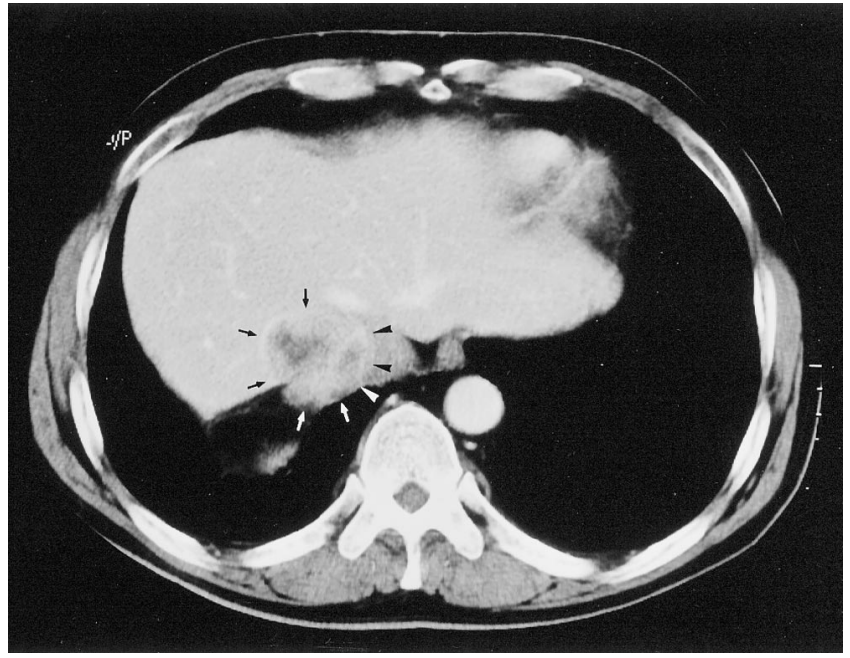

Figure 5. In this patient with a recurrent colorectal liver metastasis in the paracaval portion, computed tomography showed an unclear relationship between the tumor (arrows) and the inferior vena cava (IVC; arrowheads). At laparotomy, the wall of the IVC was involved to more than two-thirds the circumference of the vessel. Therefore, the IVC was clamped at the prehepatic portion and infrahepatically just below the confluence of the hepatic veins. The IVC was then reconstructed with a patch graft closure using Gore-Tex and remained clamped for 57 minutes. During this period, liver perfusion was maintained with outflow of the hepatic veins. Liver resection consisted of a limited resection, and liver dissection was accomplished under the Pringle maneuver (clamping time 123 minutes).

consequent hemodynamic imbalance, which should limit its application. ${ }^{5}$ Further, it could increase the amount of backbleeding from the cut surface because of the higher central venous pressure that results from the infusion of fluid ad-

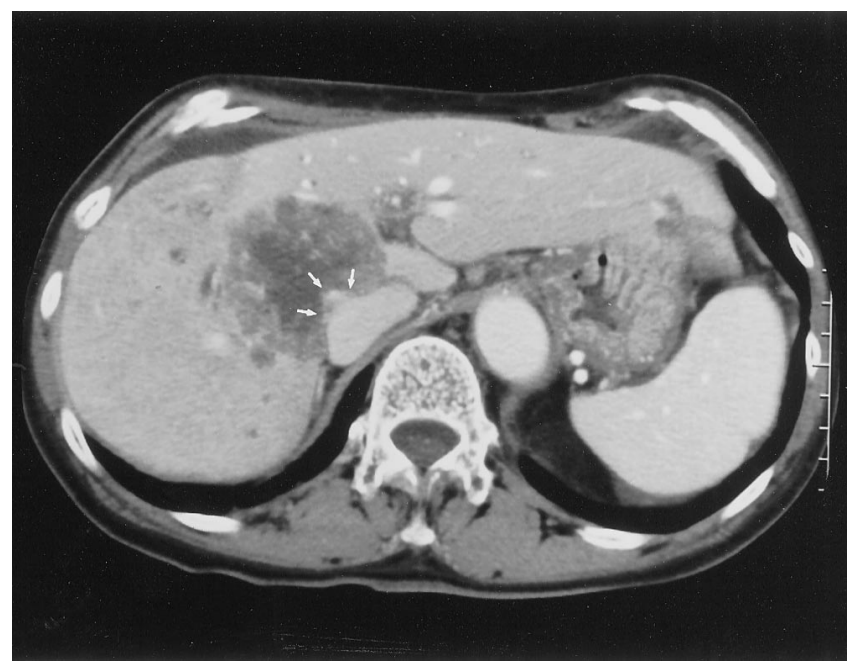

Figure 6. Computed tomography scan shows a mass-forming-type cholangiocarcinoma in the paracaval portion of the liver, which appeared to infiltrate the inferior vena cava with deformation of the vessel lumen (arrows). Although vessel infiltration was confirmed, the degree of wall involvement was less than expected and the inferior vena cava was reconstructed by direct suture. The surgical procedure consisted of extended right hepatectomy. ministered to maintain the blood pressure after releasing the aortic clamp. ${ }^{32}$ For these reasons, TVE with supraceliac aortic clamping has been almost completely abandoned.

Major advantages ascribed to TVE are the presumption that it increases the safety of liver resection, with low risk and without inducing irreversible deleterious effects on the liver ultrastructure. ${ }^{33}$ In particular, it has been reported that TVE enables a significant reduction in intraoperative blood loss and the consequent need for blood transfusion. ${ }^{5}$ However, Berney et $\mathrm{al}^{30}$ reported that $66 \%$ of patients required blood transfusions for resection of tumors in contact with the IVC or hepatic veins despite TVE. Other authors have reported that $40 \%$ to $60 \%$ of patients who underwent hepatectomy under TVE required blood transfusion. ${ }^{34-36}$ Yamaoka et al, ${ }^{37}$ in patients with and without cirrhosis who underwent major hepatectomy under TVE, reported blood loss ranging from 5,500 to $6,000 \mathrm{~mL}$. Emond et $\mathrm{al}^{38}$ reported a $0 \%$ death rate in a series of 49 patients with malignant and benign tumors who underwent liver resection under TVE; however, 55\% of them received transfusions. These transfusion rates exceed the $30 \%$ that is commonly considered acceptable. ${ }^{39}$ The use of heparin, the careless handling of blood vessels in a bloodless surgical field, and the use of a biopump (Medtronic Bio-Medicus, Inc., Eden Prairie, MN) are possible explanations for a nonnegligible amount of bleeding despite the use of TVE. ${ }^{40}$ Careless liver dissection induced by a bloodless surgical field during TVE may be responsible for some cases in which a second operation was needed as a result of rebleeding from the liver edges. ${ }^{5,31}$

In our series, TVE was sometimes considered but it was never used, even though $41.4 \%$ of the procedures were major resections, $19.5 \%$ of the patients had tumors that were in or extended to the paracaval portion of segment 1 , and $3 \%$ of the patients required reconstruction of one hepatic vein or of the IVC. The 265 patients in group B would have been treated using TVE by some surgeons, ${ }^{28,29,34,38}$ whereas the 64 patients in group A would have almost certainly undergone TVE. ${ }^{6,7}$ However, without TVE, we had a low transfusion rate (3.9\%), and this was even lower in group A (3.1\%). Further, these results were achieved with no perioperative deaths and a negligible rate of major complications.

We consider that these good results in these patients are due to wide J-shaped thoracophrenolaparotomies, accurate and meticulous liver mobilization, and liver parenchyma dissection under intermittent Pringle maneuver or selective vascular occlusion, ultrasonographic guidance, and tidal volume control.

The need for a thoracotomy is a subject of debate, but we believe that access to the chest is useful because with a J-shaped thoracophrenolaparotomy, the dissection plan is perpendicular to the surgical wound and the surgical field is just in front of the surgeon. Further, accidental bleeding from injury to the hepatic veins can be controlled easily, and the need for forced mobilization of the right lobe of the 

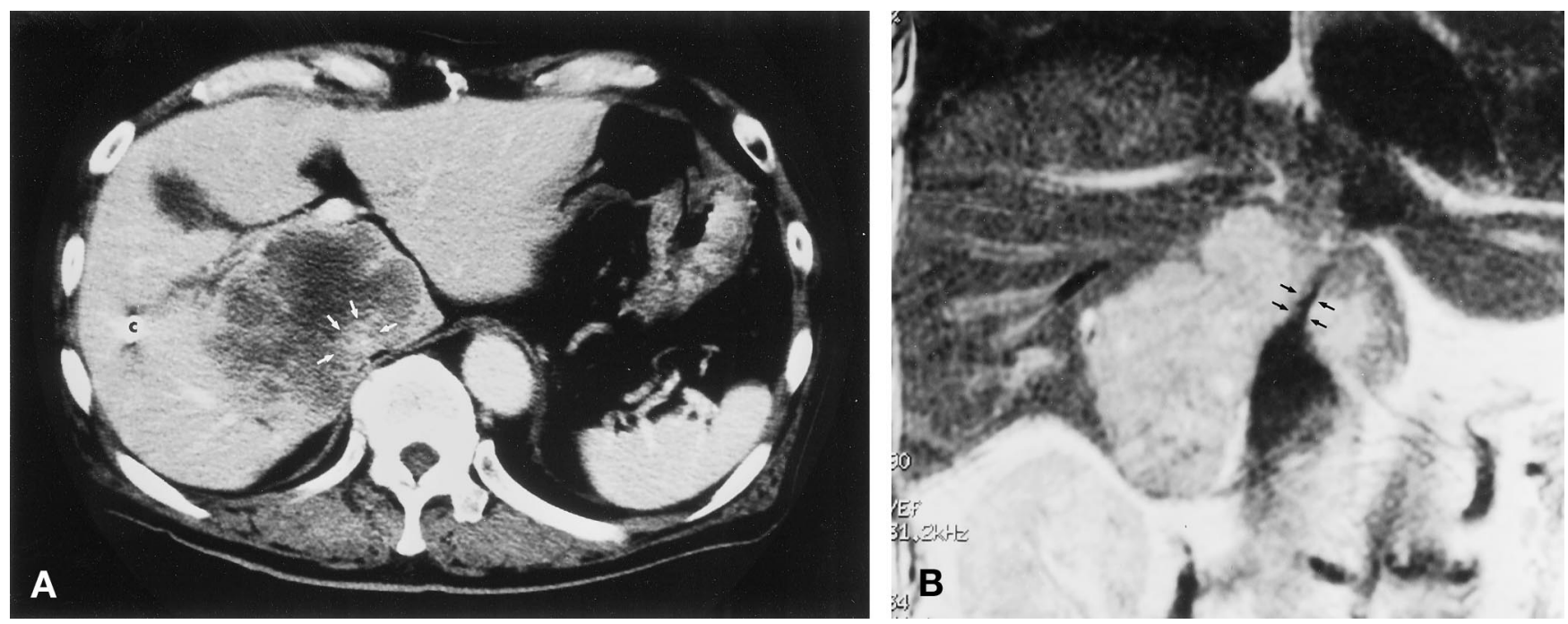

Figure 7. In this patient, the computed tomography scan (A) and the sagittal view on magnetic resonance imaging (B) apparently showed almost complete involvement of the inferior vena cava (IVC) by a colorectal liver metastasis (T). In A, the lumen of the IVC was not clear (arrows), whereas in B, the vessel caliber was markedly reduced (arrows). Despite the imaging features, at laparotomy the degree of parietal tumor infiltration was less than expected, and the IVC was reconstructed by direct suture. The liver resection consisted of extended right hepatectomy. The small c inside a high-density spot shows one of the coils inserted during the preoperative embolization of the right portal branches.

liver, which can disturb the hepatic vascular supply and rupture the hepatocellular carcinoma, is reduced. This thoracoabdominal approach makes it easier for the surgeon to place a hand posterior to the liver after dissection of the bare area and to expose the retrohepatic IVC during right-sided major liver resections. In this way, during liver parenchymal dissection the surgeon can control the patency of the right hepatic vein with a finger by clamping it when an accidental injury occurs. Moreover, the liver can be pulled up, and this reduces back-bleeding and maintains, with the aid of suction, a bloodless surgical field. Finally, the surgeon can mark and drive the direction of the dissection with the fingertips. A thoracophrenolaparotomy and a chest tube are more unpleasant for the patient, and postoperative complications such as pleural effusions, atelectasis, pneumonia, and pneumothorax may occur. In our series, these occurred in 42 patients $(13.7 \%$ ), representing $50 \%$ of the postoperative complications. However, the postoperative hospital stay in these patients was not significantly longer than that in patients who did not have these complications $(P=.08)$. Therefore, the opportunity to manage the liver better during a major resection involving the right segments and the paracaval portion of the liver justifies this approach.

Maintaining a low central venous pressure is a commonly accepted way of reducing blood loss during liver parenchymal dissection. ${ }^{41,42}$ For this purpose, we administer a muscle relaxant and reduce the tidal volume, thus limiting back-bleeding from accidentally injured hepatic veins, and reducing the need for blood transfusion. Conversely, the problem of air embolism can be easily eliminated by keeping the central venous pressure at more than $0 \mathrm{cmH}_{2} \mathrm{O}$. In this way, there is no risk of pulmonary embolism even with injury to the IVC. As confirmation, in the 329 patients in this study and in the entire series of 471 who underwent hepatectomies, there was no case of pulmonary embolism clinically evident. However, the risk of postoperative pulmonary embolism in patients who undergo TVE has not been estimated. In these patients, at least in those with associated risk factors such as obesity and advanced age, clamping of the IVC could increase the risk of postoperative deep vein thrombosis and pulmonary embolism.

Intraoperative ultrasonography is a well-recognized tool not only for better staging of liver tumors but also for performing safe liver resection. ${ }^{43}$ Accurately defining the relationship between the target lesion and the major intrahepatic vessels gives the surgeon crucial information about resectability and the most appropriate surgical approach. Intraoperative ultrasound also aids in liver dissection by guiding the surgeon's hands in the correct plane to avoid the ligation of vessels that should be preserved and if divided would increase the volume of liver to be removed. This may have also contributed to the low rate of trisectoriectomies $(1 \%)$. Finally, intraoperative ultrasound enables an immediate check of the patency of vascular grafts in the eventuality of vein reconstruction.

All these techniques must be combined with careful dissection of the liver parenchyma and with accurate hemostasis of the wall of the accidentally injured hepatic vein by means of fibrin glue and suture, which should be applied only after a wide exposure of the source of bleeding.

This policy could lead to a safe major liver surgery without the need for TVE. Conversely, in the only controlled study on this topic to date, Belghiti et $\mathrm{al}^{6}$ showed that TVE is an invasive technique with a complication rate 
2.5-fold greater than in patients treated under portal triad clamping. Side effects such as a reduction in cardiac output and central venous pressure, compensated by increased heart rate and peripheral vascular resistance, have been described. ${ }^{6,38}$

In 1985, Nagasue et $\mathrm{al}^{44}$ proposed a less-invasive form of TVE that was adopted for segmentectomies in patients with cirrhosis. It consisted of the simultaneous occlusion of hepatic inflow and outflow by the Pringle maneuver and clamping of the hepatic vein draining the segment to be removed. The authors, in comparing patients treated with this technique, those who underwent liver resection with no vascular control, and those treated using the Pringle maneuver, found significantly lower intraoperative blood loss and no deaths in patients treated by occluding the draining hepatic vein. They concluded that their technique was safe and promising. However, their experience is relatively old, and a surgical death rate of $10.5 \%$ was observed in patients treated by clamping only the portal triad, which is much higher than the mortality rates of the actual series. Nevertheless, other authors have followed this pilot study to reduce the invasiveness of TVE. Okuda et $\mathrm{al}^{45}$ proposed balloon occlusion of the hepatic veins, which seems a complex and invasive procedure, considering the need for transhepatic puncture and cannulation of the hepatic veins. Recently, Cherqui et $\mathrm{al}^{46}$ proposed again the aforementioned technique introduced by Nagasue et al. However, $30 \%$ of the patients required blood transfusion, and TVE was still considered necessary in patients with tumors in contact with the IVC. In these patients, Belghiti et al, ${ }^{6}$ who reported a higher risk of TVE, still recommend the use of TVE. Conversely, our experience, using the techniques described above, shows that major liver surgery can be performed safely without TVE in all patients, even those with a tumor in the paracaval portion of the liver. In fact, even in the presence of infiltration of the IVC, the grade of involvement of its wall by the tumor is generally less than that expected by imaging findings. The absence of significant differences in blood loss, operative length, and complication rates between patients with tumors involving the cavohepatic junction and the others in the study demonstrates the safety of surgical treatment performed without the aid of TVE, even in such patients. Such safety is again highlighted by the lower rate of transfusion among patients with tumor involvement of the cavohepatic junction.

The retrospective nature of this survey and the lack of a control group probably introduce some biases. However, these selection biases are present in most of the clinical trials on this topic, including those that highlight the advantages of TVE. Moreover, the high rate of major resections and of tumors in segment 1 , the nonnegligible number of procedures that required vascular reconstruction, and conversely the complete tumor clearance in all patients reduce the risk that patients who could have benefited from TVE were considered to have unresectable disease and then excluded from this analysis. Therefore, the results of this retrospective study reaffirm the utility of intermittent warm ischemia and suggest that TVE should be restricted to exceptional patients, such as those with infiltration of the IVC that demands substitution of the involved vessel.

\section{References}

1. Nagourney DM, Van Heerden JA, Ilstrup DM, et al. Primary hepatic malignancies. Surgical management and determinants of survival. Surgery 1989; 106:740-749.

2. Jamieson GG, Corbel L, Campion JP, et al. Major liver resection without blood transfusions: is it a realistic objective? Surgery 1992; 112:32-36.

3. Makuuchi M, Takayama T, Gunven P, et al. Restrictive versus liberal blood transfusion policy for hepatectomies in cirrhotic patients. World J Surg 1989; 13:644-648.

4. Yamamoto J, Kosuge $\mathrm{T}$, Takayama $\mathrm{T}$, et al. Perioperative blood transfusion promotes recurrence of hepatocellular carcinoma after hepatectomy. Surgery 1994; 115:303-309.

5. Bismuth H, Castaing D, Garden J. Major hepatic resection under total vascular exclusion. Ann Surg 1989; 210:13-9.

6. Belghiti J, Noun R, Zante E, et al. Portal triad clamping or hepatic vascular exclusion for major liver resection. A controlled study. Ann Surg 1996; 224:155-161.

7. Grazi GL, Mazziotti A, Jovine E, et al. Total vascular exclusion of the liver during hepatic surgery. Selective use, extensive use or abuse? Arch Surg 1997; 132:1104-1109.

8. Couinaud C. Le foie, etude anatomiques et chirurgicales. Paris: Masson; 1957.

9. Makuuchi M, Hasegawa H, Yamazaki S. Ultrasonically guided subsegmentectomy. Surg Gynecol Obstet 1985; 161:346-350.

10. Miyagawa S, Makuuchi M, Kawasaki S. Outcome of major hepatectomy with pancreatoduodenectomy for advanced biliary malignancies. World J Surg 1996; 20:77-80.

11. Torzilli G, Makuuchi M, Inoue K, et al. No-mortality liver resection for hepatocellular carcinoma in cirrhotic and non-cirrhotic patients: is there a way? A prospective analysis of our approach. Arch Surg 1999; 134; 984-992.

12. Pringle JH. Notes on the arrest of hepatic hemorrhage due to trauma. Ann Surg 1908; 48:541-549.

13. Man K, Fan ST, Ng IOL, et al. Prospective evaluation of Pringle maneuver in hepatectomy for liver tumors by a randomized study. Ann Surg 1997; 226:704-713.

14. Man K, Fan ST, Ng IOL, et al. Tolerance of the liver to intermittent Pringle maneuver in hepatectomy for liver tumors. Arch Surg 1999; 134:533-539.

15. Nagasue N, Yukaya H, Suehiro S, et al. Tolerance of the cirrhotic liver to normothermic ischemia. A clinical study of 15 patients. Am J Surg 1984; 147:772-775

16. Kim YI, Nakashima K, Tada I, et al. Prolonged normothermic ischaemia of human cirrhotic liver during hepatectomy: a preliminary report. Br J Surg 1993; 80:1566-1570.

17. Wu CC, Hwang CR, Liu TJ, et al. Effects and limitations of prolonged intermittent ischemia for hepatic resection of the cirrhotic liver. Br J Surg 1996; 86:121-124.

18. Belghiti J, Noun R, Malfosse R, et al. Continuous versus intermittent portal triad clamping for liver resection. A controlled study. Ann Surg 1999; 229:369-375.

19. Sukigara M, Makuuchi M, Omoto R. Intraoperative use of real-time two-dimensional Doppler echography for hepatectomy. Gekashinryou 1986; 9:1025-1030.

20. Takasaki K, Saito A, Akimoto S, et al. Echo guided hepatectomy. Shyokaki-geka 1985; 8:1511-1516.

21. Launois B, Jamieson GG. The importance of Glisson's capsule and its sheaths in the intrahepatic approach to resection of the liver. Surg Gynecol Obstet 1992; 174:7-10. 
22. Yanaga K, Matsumata T, Nishizaki T, et al. Alternate hemihepatic vascular control technique for hepatic resection. Am J Surg 1993; 165:365-366.

23. Yamanaka N, Furukawa K, Tanaka W, et al. Topical cooling-assisted hepatic segmentectomy for cirrhotic liver with hepatocellular carcinoma. J Am Coll Surg 1997; 184:290-296.

24. Matsumata T, Kanematsu T, Shirabe K, et al. Modified technique of Pringle's maneuver in resection of the liver. Surg Gynecol Obstet 1991; 172:245-246.

25. Myagawa S, Makuuchi M, Kawasaki S, et al. Changes in serum amylase level following hepatic resection in chronic liver disease. Arch Surg 1994; 129:634-638.

26. Miyagawa S, Makuuchi M, Kawasaki S, et al. Serum amylase elevation following hepatic resection in patients with chronic liver disease. Am J Surg 1996; 171:235-238.

27. Heaney JP, Stanton WK, Halbert DS, et al. An improved technique for vascular isolation of the liver: experimental study and case reports. Ann Surg 1966; 163:237-241.

28. Huguet C, Addario-Chieco P, Gavelli A, et al. Technique of hepatic vascular exclusion for extensive liver resection. Am J Surg 1992; 163:602-605.

29. Emond J, Wachs ME, Renz JF, et al. Total vascular exclusion for major hepatectomy in patients with abnormal liver parenchyma. Arch Surg 1995; 137:824-830.

30. Berney T. Mentha G, Morel P. Total vascular exclusion of the liver for the resection of lesions in contact with the vena cava or the hepatic veins. Br J Surg 1998; 85:485-488.

31. Stephen MS, Gallagher J, Sheil AGR, et al. Hepatic resection with vascular isolation and routine supraceliac aortic clamping. Am J Surg 1996; 171:351-355.

32. Leow CK, Lau WY, Li AKC. Hepatic resection with vascular isolation and routine supraceliac aortic clamping. Am J Surg 1997; 173:149.

33. Moussa ME, Sarraf CE, Uemoto S, et al. Effect of total hepatic vascular exclusion during liver resection on hepatic ultrastructure. Liver Transpl Surg 1996; 2:461-467.
34. Brancatisano R, Isla A, Habib N. Is radical hepatic surgery safe? Am J Surg 1998; 175:161-163.

35. Nuzzo G, Giuliante F, Giovannini I, et al. Hepatic resections in normothermic ischemia. Surgery 1996; 120:852-858.

36. So SK, Monge H, Esquivel CO. Major hepatic resection without blood transfusion: experience with total vascular exclusion. J Gastroenterol Hepatol 1999; 14(suppl):28-31.

37. Yamaoka Y, Ozawa K, Kumada K, et al. Total vascular exclusion for hepatic resection in cirrhotic patients. Arch Surg 1992; 127:276-280.

38. Emond JC, Kelley SD, Heffron TG, et al. Surgical and anesthetic management of patients undergoing major hepatectomy using total vascular exclusion. Liver Transpl Surg 1996; 2:91-98.

39. Mariette D, Smadja C, Noveau S, et al. Preoperative predictors of blood transfusion in liver resections for tumor. Am J Surg 1997; 173:275-279.

40. Hamazaki K, Yagi T, Inagaki M, et al. Hepatectomy under extracorporeal circulation. Surgery 1995; 118:98-102.

41. Rees M, Plant G, Wells J, Bygrave S. One hundred and fifty hepatic resections: evolution of technique towards bloodless surgery. Br J Surg 1996; 83:1526-1529.

42. Cunningham JD, Fong Y, Shriver C, et al. One hundred consecutive hepatic resections. Blood loss, transfusion, and operative technique. Arch Surg 1994; 129:1050-1056.

43. Makuuchi M, Takayama T, Kosuge T, et al. The value of ultrasonography for hepatic surgery. Hepatogastroenterology 1991; 38:64-70.

44. Nagasue N, Yukaya H, Ogawa Y, et al. Segmental and subsegmental resection of the cirrhotic liver under hepatic inflow and outflow occlusion. Br J Surg 1985; 72(7):565-568.

45. Okuda K, Nakayama T, Tanikawi S, et al. A new technique using an occlusion balloon catheter for the hepatic vein. Am J Surg 1992; 163:431-434.

46. Cherqui D, Malassagne B, Colau PI, et al. Hepatic vascular exclusion with preservation of the caval flow for liver resections. Ann Surg 1999; 230:24-30. 